\title{
O067. Osmophobia in allodynic migraine: role of frequency of attacks and headache duration
}

\author{
Carlo Lovati ${ }^{{ }^{*}}$, Luca Giani ${ }^{1}$, Elisa Capiluppi ${ }^{1}$, Giulia Preziosa ${ }^{1}$, Domenico D’Amico ${ }^{2}$, Claudio Mariani ${ }^{1}$ \\ From Abstracts from the 1st Joint ANIRCEF-SISC Congress \\ Rome, Italy. 29-31 October 2015
}

\begin{abstract}
Background
Migraine is a primary headache with recurrent attacks of head pain with associated symptoms like nausea, phonophobia, and photophobia. Osmophobia, although not included in diagnostic criteria, seems to be a very migrainespecific symptom [1]. Cutaneous allodynia (CA) is also a common symptom in migraine, especially when frequency of attacks is high. CA is considered a clinical manifestation of central sensitization, a mechanism involved in migraine chronification [2]. Recent works put in evidence a relationship between the presence of osmophobia and CA in migraineurs [3]. This study was aimed to investigate possible clinical elements able to influence the relationship between osmophobia and allodynia in migraine.
\end{abstract}

\section{Materials and methods}

We enrolled 871 patients consecutively evaluated in our Headache Center. Chronic migraine was defined as a mean frequency of headache of at least 15 days per month. Two hundred and sixty-three patients had chronic migraine (63 with aura, ChMA, and 200 without aura, ChMO) and 608 were episodic (165 with aura, MA, and 443 without aura, $\mathrm{MO})$.

\section{Results}

Osmophobia was significantly more frequent among patients with CA with respect to patients without CA ( $33.9 \%$ vs $26.7 \%, p=0.016$ at Chi square test). The association between these two symptoms was significant only in chronic migraineurs, among which osmophobia was present in $39.4 \%$ of allodynic patients and in $24.1 \%$ of non-allodynic patients ( $\mathrm{p}=0.008$ at Chi square test). No difference was found in the distribution of osmophobia comparing chronic migraineurs with and without aura

\footnotetext{
* Correspondence: lovati.carlo@hsacco.it

${ }^{1}$ Headache Center, Neurology Unit, A.O. L. Sacco, Milan, Italy

Full list of author information is available at the end of the article
}

(44\% of ChMA and 37\% of ChMO). Both CA and osmophobia were significantly more frequent among women with respect to men: CA was found in $30 \%$ of men and $55 \%$ of women $(\mathrm{p}<0.001)$ and osmophobia in $18.2 \%$ of men and $32.6 \%$ of women $(\mathrm{p}=0.001)$. The relationship between $\mathrm{CA}$ and osmophobia was confirmed in both ChMA and ChMO among women. Even if the proportion was similar, significance was not found among men, probably because of the smaller sample size. Osmophobic episodic migraineurs, both with and without aura, had a longer migraine history. This evidence was not found among chronic patients.

\section{Conclusions}

The highlighted relationship between allodynia and osmophobia seems not to be influenced by gender nor by aura. The observation that it is related to a higher frequency of attacks and longer history of migraine may be interpreted as a common consequence of central sensitization, able to induce in parallel a distortion of both cutaneous sensitivity (CA) and olfaction (osmophobia).

Written informed consent to publication was obtained from the patient(s).

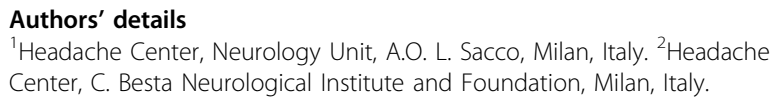

Published: 28 September 2015

References

1. Baldacci $F$, et al: Clinical features associated with ictal osmophobia in migraine. Neurol Sci 2015, 36(1):43-6.

2. Louter MA, et al: Cutaneous allodynia as a predictor of migraine chronification. Brain 2013, 136(Pt 11):3489-96.

3. Lovati $C$, et al: Osmophobia in allodynic migraineurs: cause or consequence of central sensitization? Neurol Sci 2015, 36(Suppl 1):145-7.

doi:10.1186/1129-2377-16-S1-A104

Cite this article as: Lovati et al.: O067. Osmophobia in allodynic migraine: role of frequency of attacks and headache duration. The Journal of Headache and Pain 2015 16(Suppl 1):A104. 